Review

\title{
The Sex Chromosomes of Frogs: Variability and Tolerance Offer Clues to Genome Evolution and Function
}

\author{
Jacob W. Malcom, Randal S. Kudra, and John H. Malone ${ }^{凶}$ \\ Department of Molecular and Cell Biology, University of Connecticut, Storrs, 06269 USA \\ $\triangle$ Corresponding author: Phone: 860-486-1899; Fax: 860-486-1936; Email: john.malone@uconn.edu \\ (C) Ivyspring International Publisher. This is an open-access article distributed under the terms of the Creative Commons License (http:/ / creativecommons.org/ \\ licenses/by-nc-nd/3.0/). Reproduction is permitted for personal, noncommercial use, provided that the article is in whole, unmodified, and properly cited.
}

Published: 2014.03.20

\begin{abstract}
Frog sex chromosomes offer an ideal system for advancing our understanding of genome evolution and function because of the variety of sex determination systems in the group, the diversity of sex chromosome maturation states, the ease of experimental manipulation during early development. After briefly reviewing sex chromosome biology generally, we focus on what is known about frog sex determination, sex chromosome evolution, and recent, genomics-facilitated advances in the field. In closing we highlight gaps in our current knowledge of frog sex chromosomes, and suggest priorities for future research that can advance broad knowledge of gene dose and sex chromosome evolution.
\end{abstract}

Key words: Homomorphic, aneuploidy, dosage, compensation, sex determination

\section{Introduction}

Changes in gene copy number are a critical source of variation for evolution, but new copy numbers that alter gene dose often presents a challenge for organismal survival. The scale of copy-number change can range from the deletion or duplication of one or a few genes, to polyploidization of all genes through whole-genome duplication [1-3]. Post-duplication, genes typically have one of three fates: nonfunctionalization, in which only one of the copies retains any function; subfunctionalization, in which the copies diversify to "share" the function of the original; or neofunctionalization, in which one of the gene copies gains a novel function $[2,4,5]$. There is a strong population genetic component to the probability of these different fates, but before the fate is realized, the organism containing the duplicates faces the challenge of surviving with an over-abundance of gene product for duplicate genes or under-abundance of non-duplicated, interacting genes [6-10]. This gene dose problem can threaten the function of a cell or an entire organism because of the integrated nature of molecular function, a concept central to the "genome balance hypothesis" [11].

Despite the importance of gene dose in basic biological function, we know relatively little about the mechanisms that regulate this process, why dose changes are often fatal, or why some can be tolerated. In contrast to the general problem of gene dose that accompanies aneuploidy, the presence of different sexes within a species-which occurs in nearly all 66,000 vertebrate species and an unknown number of invertebrates - represents a naturally occurring system with extensive gene imbalance. Diverging sex chromosomes are an ideal system to study genome balance and gene dose because males and females are viable, one sex or the other is often aneuploid across a chromosome, and genomes have adapted to handle these dosage differences. Sex chromosomes are natu- 
rally highly variable, with extensive differences in sex determining systems and sex chromosome morphology between and within species [12]. Thus, by studying the form and function of naturally occurring aneuploidy in the form of sex chromosomes, we can leverage evolutionary variation and diversification to gain insight into normal function as a reference against which dosage dysfunction is compared. Beyond addressing fundamental problems in evolution, this powerful system has advantages for studying human disease, e.g., many human diseases are caused by gene dose variation [13-19], and understanding how evolution "solves" the dose problem could provide insight that guides the development of disease treatments.

Here we briefly review several aspects of sex chromosome biology, from flies to humans. Many attributes of frog evolution and sex chromosomes offer an ideal opportunity to address the problem of gene dose tolerance, which we review in greater detail. We close with what we think are the necessary next steps in developing frogs as a model system for advancing our understanding of the causes and consequences of gene dose variation.

\section{A Brief Introduction to Sex Determina- tion and Gene Dose}

A broad look at sex chromosomes and associated gene dose challenges has recently been presented [20]; here we briefly review the topic to set the stage for our focus on frogs.

The differentiation of sexes can be driven by a variety of mechanisms. In many fish and reptiles, the sex determination cascade is triggered by differences in the external environment [21]; in other fish and reptiles, and all amphibians, birds, and mammals, sex is encoded by a genetic locus that segregates with one sex only [22]. Genetic sex determination thus removes an element of chance by guaranteeing the production of females and males, but as a consequence, represents a system with imbalanced gene dose between males and females. Genetic sex determination can be monogenic or polyfactorial, distinctions that are correlated with the degree of heteromorphism in sex chromosomes [23]. In most mammals the male sex is determined genetically by the Y-linked SRY locus [24-26]. In teleost fish [27], Drosophila melanogaster [28-30], and Caenorhabditis elegans [31], DM domain-containing genes have been implicated in sex differentiation. In multiple vertebrates, including fish, amphibians, turtles, alligators, birds, and mammals the gene DMRT has been shown to be needed in gonadal development [27, 32-36].

The presence of a sex determining gene on a chromosome defines the sex chromosome and gives rise to several genetic sex determination systems. Perhaps the best-known is $\mathrm{XY}$ sex determination, where males are heterogametic $(X Y)$ and females are homogametic $(X X)$; $X Y$ sex determination is found in insects, fish, reptiles, frogs, and mammals. Fruit fly (Drosophila) sex determination is XY-based, but occurs in a different fashion: males are heterogametic $X Y$, but the ratio of $X$ chromosomes to autosomes determines the initial sex $[37,38]$. The second major sex determination system is ZW-based, males are the homogametic sex (ZZ), and is found in fish, reptiles, frogs, birds, and some insects. In addition to the two most well-studied systems there are a variety of alternative $\mathrm{X}$ - and $\mathrm{W}$-based sex determination systems, such as $\mathrm{XO}$ in C. elegans and many insects [39] and $\mathrm{OW}$ in some frogs [40].

Sex chromosomes differ in the number of copies in a population ( $\mathrm{Y}$ or $\mathrm{W}=1 / 4$ the effective population size of autosomes) and therefore have different population genetic dynamics than autosomes. One result of this difference is a divergence in sex chromosome morphology and elimination of recombination: young sex chromosomes are homomorphic (same size), but gradually the $\mathrm{Y}$ and $\mathrm{W}$ shrink relative to $\mathrm{X}$ and $\mathrm{Z}$, recognized as heteromorphic. This is thought to occur primarily through Muller's Ratchet, which highlights that deleterious mutations accumulate in non-recombining genomes or genomic regions [41]. Because deleterious mutations accumulate through time, rather than being purged by recombination, $\mathrm{Y}$ and $\mathrm{W}$ chromosomes gradually lose genes as they mature, creating a gene dose problem [42]. Beyond the evolution of sex chromosome gene content and the effects on dosage, there is the potential for an interesting interaction between the population genetics of sex chromosomes and mating systems. Specifically, we expect sexually antagonistic genes to increase on the chromosome containing the sex determining gene [43-45], but this chromosome also features a smaller effective population size and thus requires stronger selection to overcome drift. In the context of male-antagonistic mating systems with $X Y$ determination, this could accelerate the rate of $Y$ degeneration [46]; in a ZW system this could accelerate the rate of $\mathrm{W}$ chromosome degeneration. These population genetic and mating system interactions would then shape (or be shaped by) the rate of adaptation to dosage changes.

A consequence of evolving sex chromosome morphology is variation in the number of copies of sex-linked genes in males and females, leading to an expectation of large gene dose imbalances in one sex. In light of our knowledge that dose differences in 
even a single gene can be highly detrimental, how do organisms handle dosage of a large number of genes? At its extreme there may be complete sex chromosome aneuploidy, such as in flies, where the Y chromosome is devoid of genes [46]; even at intermediate stages of sex chromosome maturity, aneuploidy may span hundreds or thousands of genes.

Two major mechanisms are known to have evolved for dosage sensitivity: dosage compensation and dosage tolerance. Dosage compensation is the adjustment of gene expression to compensate for aneuploidy, and historically has been thought only to exist with sex chromosomes [47]. In eutherian mammals, dosage compensation occurs through $X$-inactivation: the ncRNA XIST suppresses most expression on one $X$ copy in females $[48,49]$. In contrast to mammals, Drosophila have a macromolecular complex thought to increase expression of genes on the single male $\mathrm{X}$ chromosome by about 2 -fold, thus making male and female expression effectively the same; it is worth noting, however, that complex network dynamics and transcriptional control are also involved [50-53]. Other compensation systems, such as incomplete and gene-specific inactivation, have been found in the monotreme platypus and chickens (an "atypical" XY system and ZW, respectively; [54]).

A curious situation arises when females are the heterogametic sex (ZW), such as in birds, snakes, and some fish, reptiles, frogs, and insects: dosage compensation appears to be partial, if it occurs at all (reviewed in [12, 20]). Furthermore, where partial dosage compensation mechanisms have been studied, they appear to be fundamentally different than "typical" XY sex determination systems [54]. Transcriptome analyses in several birds, liver flukes, and snakes all suggest a lack of expression adjustment on the $\mathrm{Z}$ chromosome even though the $\mathrm{W}$ chromosome is highly degenerate [20, 55-58]. The different dose mechanisms between $X Y$ and $Z W$ systems suggest different tolerance capacity to aneuploidy.

\section{Frog Sex Chromosomes}

The preceding review, although brief, raises the question, What strengths do frogs provide given the variety of sex determination systems, sex chromosome morphologies, and dosage studies in other organisms? Mammals and birds appear to be entirely $\mathrm{XY}$ and $\mathrm{ZW}$, respectively, so there is little opportunity to apply a comparative approach between closely related species to examine how the mechanisms of compensation or tolerance evolve. Fish, amphibians, and reptiles, however, possess a variety of sex determination systems and each have particular features worthy of study. Fish are diverse with both environ- mental and genetic sex determination and homomorphic sex chromosome that are relatively new [59]. Reptiles possess a variety of sex determination systems and sex chromosome morphologies, but lack strong genetic experimental tools. Frogs possess diverse sex determination systems and sex chromosome morphologies, several other interesting chromosomal characteristics, and they are amenable to numerous experimental possibilities for the study of development, genome dynamics, and sex phenotype. That is, frogs bring the power of both evolutionary diversification and experimental manipulation to the challenge of understanding gene dose.

\section{Sex Determination}

While sex-determining genes have been found in other taxonomic groups, only a single sex determination gene has been identified in a frog. $D M-W$, an apparent paralog of DMRT1, is found on the X. laevis $\mathrm{W}$ chromosome and is sufficient to cause development of female phenotypes [36]. Curiously, $D M-W$ does not occur in the closely related $X$. tropicalis and therefore there must be a different gene responsible [60]. This and examples from fish suggest that gene duplication and subsequent sub- or neofunctionalization may play an important role in sex determining gene evolution. Rather than alternate alleles, another possible sex determination mechanism may arise from dosage differences of a sex determining gene. If frogs can handle differences in sex determining gene dosage, then sex may be determined in a manner akin to haploinsufficiency, where a single copy in females $(\mathrm{ZW})$ or males $(\mathrm{XY})$ is insufficient to trigger the cascade producing males (ZZ) or females (XX). This is the proposed mode of action of Z-linked DMRT1 in chickens [61].

Above the level of individual sex determination genes are sex determination systems. Like invertebrates, reptiles, and fish, amphibians exhibit extensive diversification of sex determination systems, including $X Y$ and $Z W$, and $0 \mathrm{~W}$; all of these are present in frogs (examples of $X Y$ and $Z W$ species provided in Figure 1). Furthermore, there is extensive sex determination system switching between species and clades, in particular a bias of ZW to XY switching [60, 62]. The pattern of sex determination switching carries with it a host of interesting implications in light of the need for repeated adaptation to dosage change and interactions with the effects of sexual selection in $X Y$ versus $\mathrm{ZW}$ systems. One of the most intriguing and potentially powerful patterns of frog sex determination evolution is that sex determination systems change both between and within frog lineages $[63,64]$. As a result, frogs can be used for comparative tests not 
only between closely related species, such as Pristmantis or Gastrotheca, but even within species. In Rana temporaria and R. rugosa, for example, frogs of different sex determination systems can occur in the same population and interbreed; a candidate explanation for the observed pedigrees is that multiple sex chromosome combinations map to a single phenotypic sex [64-66]. Frogs thus provide a range of variation needed to examine the effects of sex determination system on gene dosage.

In addition to the diversity of sex determination systems there is variation in sex chromosome morphology in frogs. Some $95 \%$ of frog species examined to-date, including Xenopus, the current model frog, possess homomorphic sex chromosomes $[67,68]$. Why and how sex chromosome homomorphism is maintained in so many species of this (often evolutionarily ancient) group is a mystery. Two current hypotheses, each with support in different groups of frogs, aim to explain the pattern. The first, termed the "fountain of youth" hypothesis, posits that even rare sex chromosome recombination, e.g., during a bout of sex rever-

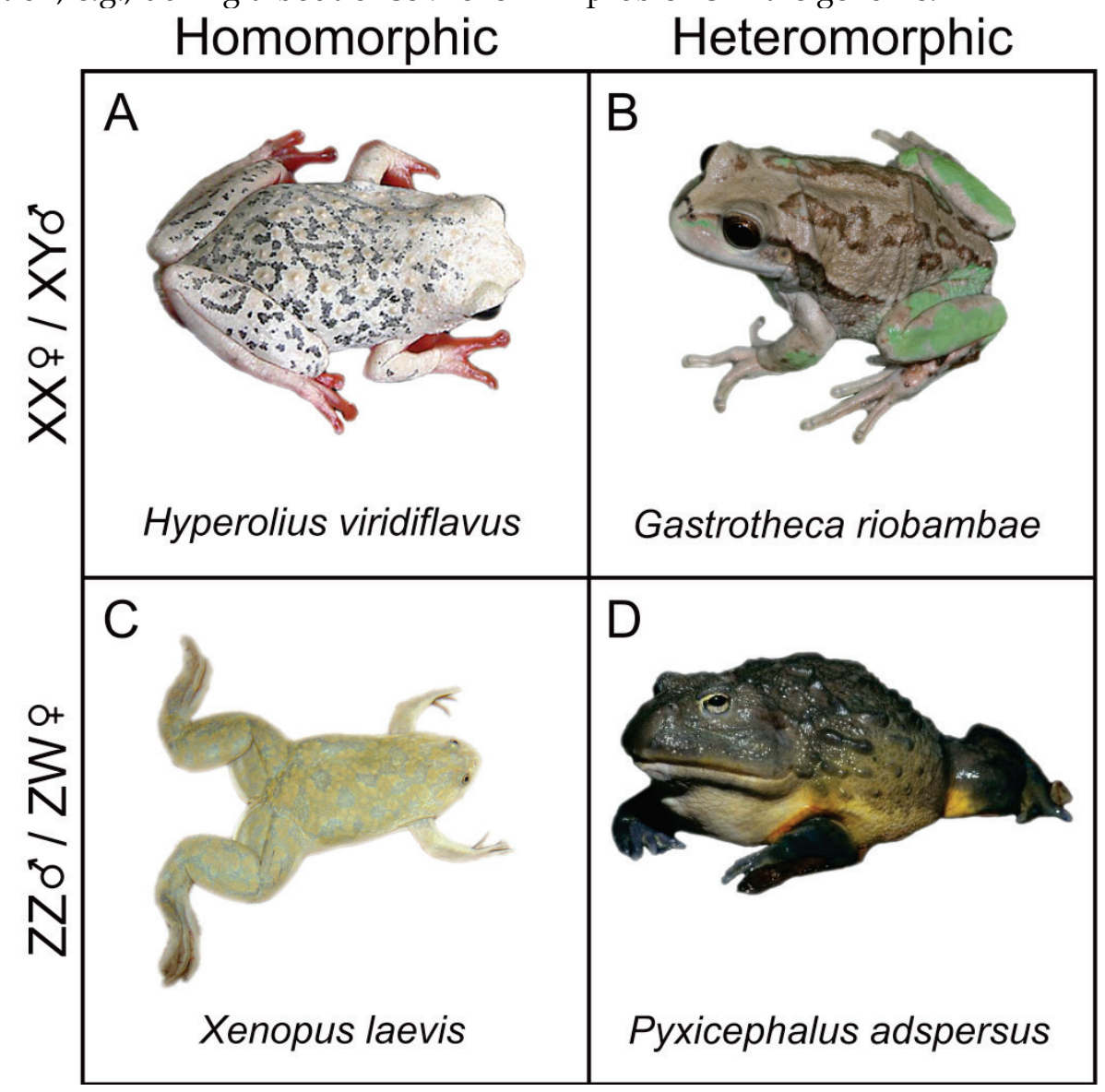

Figure I. Representative frogs from $X Y$ and $Z W$ sex determination systems. (A) $H$. vividiflavus features $X Y$ sex determination with homomorphic sex chromosomes; interestingly, females of the species have bright coloration. (B) G. riobambae is an example of $X Y$ sex determination with heteromorphic sex chromosomes; oddly, $Y$ chromosome in males is larger than the $X$ chromosome (image $(C)$. Janzen, adapted with permission). (C) The model species $X$. laevis is a ZW sex determination system with homomorphic sex chromosomes, which contrasts with (D), P. adspersus, which is ZW with heteromorphic sex chromosomes.

sal [69], allow genetic diversity and homomorphy to be maintained. This hypothesis is supported in several European tree frogs and several diploid toads (Hyla and Bufo; [70-72]). The second hypothesis, termed the "high turnover" model, posits that the identity of the sex chromosomes evolves rapidly as mutations affecting sex determination occur on autosomes, effectively changing which is the sex chromosome before Muller's Ratchet can act to degrade the $Y$ or $W$ [73]. The high turnover model is supported with data from Ranids and Bufonids, broad scale phylogenetic analyses, and interspecific cross data, which all suggest that turnovers between chromosomes to produce new sex chromosomes are common [60, 62-64, 74-76]. Which mechanism, the fountain of youth, or chromosome turnover is the more influencing mechanism for sex chromosome homomorphy is an intriguing question, and given that $95 \%$ of frog species have homomorphic sex chromosomes points to the importance of dealing with the gene dosage issue because homomorphism is one way to eliminate dose problems in the genome. 
One idea that could resolve the paradox of gene dosage and sex chromosome evolution is that particular genomic regions-e.g., ones that harbor genes related to sex determination-might more easily become sex chromosomes than other genomic regions [77]. Support for this idea has come from a recent comparative genomics analysis that compared sex-linked molecular markers from deeply divergent lineages of frogs with the genome sequence of Xenopus tropicalis [78]. Their results showed extensive conserved synteny of a sex chromosome across frogs that represent 210 million years of evolution, suggesting either an ancestral chromosome that remained homomorphic for excessive evolutionary time, or the more likely scenario, that the same genomic regions have been coopted independently for sex chromosome function. An extension of this hypothesis is that these independently derived chromosomal regions may harbor dose insensitive genes. Consider a novel sex determining locus that can arise in one of two locations in the genome, one that is characterized by largely dose-sensitive genes and one characterized by largely dose-insensitive genes: the immediate fitness cost should be higher if the new locus is in the dose-sensitive region. If genome content organization is structured by dose sensitivity, then certain regions should have greater ability to tolerate sex chromosome evolution.

These recent studies have revealed many exciting new ideas for how sex chromosome homomorphy might be maintained. Indeed, homomorphy is the most abundant state of sex chromosome morphology in all animals, yet most theory has focused on explaining the more rare, but initially highly deleterious heteromorphic state, where the genome suffers a gene dose problem over time. In contrast to the majority of species that have homomorphic sex chromosome, frog species such as Pyxicephalus adspersus (ZW) and several others possess clearly heteromorphic sex chromosomes [79]. Because frogs have different sex determination systems and different states of sex chromosome evolution, comparative genomics applied to homomorphic and heteromorphic $\mathrm{XY}$ and $\mathrm{ZW}$ frog species provides a natural experiment to test for turnovers. Furthermore, this natural experiment may provide the context needed to understand the characteristics of gene dose sensitivity that might predispose some regions to be tolerant to changes in gene dose. In general, understanding why frogs with heteromorphic sex chromosomes are exceptions to the rule of sex chromosome homomorphy will provide critical insights into the mechanisms and evolution of gene dose tolerance or compensation.

\section{Mating systems}

Given the importance of understanding the evolutionary origins and dynamics of frog sex chromosomes and the idea that variation in mating systems could contribute to morphological change, it is instructive to consider the role frogs could play in testing relevant hypotheses. The first observation is that most frog species' mating systems are driven by male antagonism, through calling, fighting, and female choice [80]. This general pattern appears to hold regardless of the sex determination system, because in most frog species males call to attract females. As with sex chromosome morphology, however, there are potentially informative exceptions to the rule. For example, the African reed frogs (Hyperolius) feature at least one species (H. argus) and a species complex $(H$. viridiflavus) in which females are vibrantly colored and males are dull; another frog, Dyscophus antongilii, features large, red females and diminutive, dull males. These examples may indicate species in which female antagonism drives sexual selection, and depending on the sex determination system, may provide tests of the role of mating system in sex chromosome evolution. One of the many (sub-) species of the $H$. viridiflavus complex possesses $X Y$ sex determination and homomorphic sex chromosomes [81], but the sex determination system and sex chromosome morphology of the other species is unknown at this time. The genome size of several Hyperolius is $\leq 5 \mathrm{~GB}$ (www.genomesize.com), which is small enough to enable sequencing and assembly (see Future Directions, below) for further investigation of the relationship between sexual selection, mating system, sex determination system, and gene dosage.

\section{Dosage Tolerance and Compensation}

The presence of heteromorphic sex chromosomes, whose evolution may be shaped by sexual selection and the particulars of mating systems, suggests a need either for gene dosage tolerance or for dosage compensation. Identifying the patterns of tolerance and compensation across frog species with $X Y$ versus $\mathrm{ZW}$ sex determination, and heteromorphic versus homomorphic sex chromosomes, will be invaluable in the search for underlying mechanisms. This presents both a challenge and an opportunity because dosage compensation of frog sex chromosomes has not been well studied.

One of the few ways in which gene dosage has been examined in frogs is through the presence of Nucleolus Organizing Regions (NOR). NORs have been identified on the X chromosome of Gastrotheca riobambae $[82,83]$ and on the homomorphic $\mathrm{Z}$ chromosome of Buergeria buergeri [84]. Follow-up experi- 
ments with $B$. buergeri to directly test for dosage compensation revealed neither Barr body formation in males, nor signs of other dosage compensation mechanisms to deactivate one of the NOR copies. Correspondingly, the ribosomal RNA levels of the male frog were twice as high as the female [85-88]. These factors suggest that no dosage compensation occurs in B. buergeri and support the idea that there is at least partial dose insensitivity. Dose insensitivity may also be indicated by the presence of a NOR on some female Pyxicephalus adspersus $\mathrm{W}$ chromosomes. The NOR of P. adspersus is on chromosome pair 6 , but females can have an additional ectopic copy on the $\mathrm{W}$ chromosome $[68,79]$. In contrast to $P$. adspersus, the frog Physalaemus ephippifer possesses two NORs on the $\mathrm{W}$ chromosome and one on the homomorphic Z [89]. Gene dosage compensation in this species would then require targeting a single $\mathrm{W} N \mathrm{NOR}$ or the sole $\mathrm{Z}$ NOR. While this suite of dosage compensation mechanisms is possible in frogs, until further evidence is gathered the simplest explanation is that, as in B. buergeri, regions of frog genomes that become sex-linked may be particularly dose tolerant.

There is additional evidence that suggests frogs are dose insensitive in the form of viability in the face of aneuploidy. One species with an altered diploid number is Strabomantis biporcatus, in which $\mathrm{XY}$ males feature a Y-to-autosome fusion that results in a chromosome count of 35 , in contrast to female $2 n=36$ $[90,91]$. Another example is Leiopelma hochstetteri, in which males have no sex chromosomes while females possess a W [40]. Xenopus is a remarkable group in which ploidy level varies between species-from diploid to dodecaploid - that can be crossed in the laboratory [92].

The presence and abundance of sex chromosome heterochromatin could play an important role in shaping dose regulation. Heterochromatin composition varies between frog species and often in remarkable ways. Two species, Pristimantis euphronoides and $P$. shrevei, possess giant $\mathrm{W}$ chromosomes that in fact are the largest chromosomes in the genome, and stain heavily for constitutive heterochromatin [68, 93]. In Pyxicephalus adspersus the small $\mathrm{W}$ chromosome is characterized by partial heterochromatin, while in Gastrotheca walkeri the small Y chromosome contains no heterochromatin. Even within the same species the extent of heterochromatin can vary substantially: in $G$. pseustes, for example, the $Y$ chromosome contains no heterochromatin in some males but the $\mathrm{Y}$ has an abundance of heterochromatin in other males [94, 95]. In addition to the role that constitutive heterochromatin can play in shaping gene dose, facultative heterochromatin-such as Barr body formation in mammal $X$ dosage compensation-could play a role in frogs. Given the hypothesis that XY sex determination requires compensation, one hypothesis is that some $X Y$ frogs may be found to use sex chromosome-wide facultative heterochromatin mechanisms to regulate dosage. Below the level of entire chromosome (or chromosomal region) heterochromatin effects, gene-specific dosage regulation may be mediated by local histone methylation in frogs. Relatively little is known about frog epigenetics at this time (but see [96]), however, and no work we have found focuses on the role of methylation in frog gene dosage and sex chromosome evolution.

\section{Future Directions and Summary}

Sex chromosomes present organisms with a complex gene dosage problem over time, but the challenge is consistently met so that both sexes, regardless of sex determination system, are fully functioning and viable. How can this happen? Why does it appear that $X Y$ sex determination requires dosage compensation while $\mathrm{ZW}$ permits dose tolerance? With their diversity of sex determination systems, variation in sex chromosome morphologies, and ease of experimental manipulation, frogs are an ideal group to address these and related questions. This, in turn, provides a mechanistic link to classic problems in evolutionary biology that revolve around the important processes of gene duplication and gene loss. A practical question is then, which research efforts will prove most fruitful and provide a strong foundation for fully utilizing frog evolutionary diversity to address fundamental gene dose questions in biology?

\section{Future Directions}

While genome sequencing once required huge collaborations and concomitantly large budgets, de novo sequencing projects are now possible for individual labs. The size of many frog genomes means that a suite of genome references can readily be developed, and thereby advance our understanding of genome evolution, in particular the evolution of sex chromosomes. With genome sequences in which sex chromosomes are identified, we can examine patterns of synteny between frogs with $\mathrm{XY}$ and/or ZW systems to understand the origins and dynamics of sex chromosome evolution. The gene content of frog sex chromosomes is largely unknown at this time, much less the evolutionary origins of these genes. When genome references are coupled with gene models (supported by transcriptome research, discussed below), we can address questions such as: Are there systematic differences in the content and evolution of the $\mathrm{X} / \mathrm{Y}$ and $\mathrm{Z} / \mathrm{W}$ chromosomes? What about gene 
content differences between species with homomorphic versus heteromorphic sex chromosomes? Are proto-sex chromosomes characterized by dose-insensitive genes so that acquiring a novel sex determining locus is more likely? Is sex chromosome evolution constrained such that the same genes, genes with similar function, or genes with similar dose sensitivity repeatedly end up on sex chromosomes when the determination systems evolve independently? Beyond these sex chromosome-specific questions, the presence of genome references for a variety of frog species would be invaluable resources for more general understanding of genome evolution, the genomic basis of variation within and between species, and a host of other questions.

Beyond discovering and describing variation in genome content, our understanding of the biology of gene dose and genome balance is informed by quantifying the expression of sex-linked genes relative to autosomal genes. Here, the availability of well-refined transcriptomes to complement genome references will be very useful, and transcriptomes derived from a variety of RNA-Seq methods are a necessary component of defining gene content. Evidence to-date suggests systematic differences in dosage sensitivity between organisms with $\mathrm{XY}$ versus $\mathrm{ZW}$ sex determination; frogs, with their mixture of these sex determination systems, offer a prime test of this hypothesis. As references are developed for closely related species with different sex determination systems, and gene expression studies are conducted, we will be able to test a variety of hypotheses for sex chromosome evolution. Furthermore, a phylogenetically informed approach to frog sex-linked gene expression will allow us to evaluate if and when expression evolution, and potential dosage compensation, occur.

In addition to sequence variation - in the form of gene copy number, the gene content of sex chromosomes (and autosomes), and similar characteristics-epigenetic variation is known or thought to play a significant role in gene dose regulation. The example of Pristimantis noted earlier, in which $\mathrm{W}$ chromosomes are giant in size and almost entirely heterochromatic, suggests either (or both) limited access by transcriptional regulators and/or highly repetitive, non-functional sequences on the W. Beyond the constitutive heterochromatin as in the Pristimantis example, facultative heterochromatin differences between sexes such as variation in methylation patterns, could play an important role in gene dose dynamics. Following on genome and transcriptome sequencing of a variety of frogs with a range of sex determination systems, chromatin immunoprecipitation (ChIP) sequencing would provide essential insights into the factors affecting gene dose regulation. ChIP-Seq with both broad (e.g., histones) and specific targets (e.g., transcription factors; [97]) would provide needed insight into the role that chromatin plays in gene dose regulation between frog sexes. Comparing patterns of heterochromatin between $\mathrm{XY}$ and $\mathrm{ZW}$ frog systems, in the context of gene dose variation of sex-linked genes, may illuminate non-genetic mechanisms that are generally important to disease-causing gene dosage problems.

We noted in passing that periodic sex reversal episodes could contribute to the maintenance of frog sex chromosome homomorphy (through the "fountain of youth"), but this only hints at one of the most useful aspects of frogs in sex chromosome and gene dosage research: we can experimentally manipulate frog sex in the laboratory. Two primary mechanisms have been found for sex manipulation: pressure or temperature manipulation, and hormone treatment. These experimental manipulations create frogs of one genotypic sex that is the opposite of the phenotypic sex [98-100]. Complementing steady-state gene expression and chromatin immunoprecipitation assays with sex-reversal experiments will be a powerful way to disentangle the roles of phenotypic and genotypic sex effects on gene dose. For example, if dosage compensation is found associated with male heterogamety, then perhaps ZW males might reveal signs of dosage compensation. Additionally, reversing the phenotypic sex can help refine identification of sex determination genes by examining expression of candidate genes with respective phenotypic sex while inverting chromosome content. If a $\mathrm{W}$ chromosome gene is the trigger for female normal differentiation, then in sex-reversed males, this trigger gene should not be expressed.

\section{Summary}

Unraveling the causes and consequences of gene dose sensitivity is a challenging problem in contemporary biology that feeds into our understanding of genome evolution, but is a tractable problem if we capitalize on sex chromosomes. Even with sex chromosomes as a good candidate, the current lack of suitable model systems is problematic. Frogs and their sex chromosomes possess diverse sex determination systems and sex chromosome morphology; many species have relatively small genomes $(<5 \mathrm{~GB})$; and ease of experimental manipulation means that the genomic systems can be systematically perturbed. We are now at a point where technological and conceptual advances permit rapid development of genomic resources for systems that are ideally suited to addressing specific biological questions. Our review of 
frog sex chromosomes highlights that our slimy friends are ideal for investigating sex chromosomes and the pattern and process of dosage tolerance, which is intricately related to our understanding of human health and broad problems in evolutionary biology.

\section{Competing Interests}

The authors have declared that no competing interest exists.

\section{References}

1. Ohno S. Evolution by Gene Duplication. Berlin, Germany: Springer-Verlag; 1970.

2. Lynch M. The Origins of Genome Architecture. Sunderland, USA: Sinauer Associates; 2007.

3. Schrider DR, Hahn MW. Gene copy-number polymorphism in nature. Proc R Soc B Biol Sci. 2010; 277:3213-3221.

4. Zhou Q, Wang W. On the origin and evolution of new genes--a genomic and experimental perspective. J Genet Genomics. 2008; 35:639-648.

5. Lynch M, Force A. The Probability of Duplicate Gene Preservation by Subfunctionalization. Genetics. 2000; 154:459-473.

6. Wagner A. Genetic redundancy caused by gene duplications and its evolution in networks of transcriptional regulators. Biol Cybern. 1996; 74:557-567.

7. Malone JH, Cho D-Y, Mattiuzzo NR, et al. Mediation of Drosophila autosomal dosage effects and compensation by network interactions. Genome Biol. 2012; 13:r28.

8. Amoutzias GD, Robertson DL, Oliver SG, et al. Convergent evolution of gene networks by single-gene duplications in higher eukaryotes. EMBO Rep. 2004; 5:274.

9. Wagner A. Evolution of gene networks by gene duplications: a mathematical model and its implications on genome organization. Proc Natl Acad Sci. 1994; 91:4387-4391

10. Conant GC. Rapid reorganization of the transcriptional regulatory network after genome duplication in yeast. Proc R Soc B Biol Sci. 2010; 277:869-876.

11. Birchler JA, Veitia RA. Gene balance hypothesis: connecting issues of dosage sensitivity across biological disciplines. Proc Natl Acad Sci USA. 2012; 109:14746-14753.

12. Bachtrog D, Kirkpatrick M, Mank JE, et al. Are all sex chromosomes created equal? Trends Genet TIG. 2011; 27:350-357.

13. Lejeune J, Gautier M, Turpin R. Etude des chromosomes somatiques de neuf enfants mongoliens. Comptes Rendus Hebd Séances Académie Sci. 1959; 248:1721-1722.

14. Jacobs PA, Strong JA. A Case of Human Intersexuality Having a Possible XXY Sex-Determining Mechanism. Nature. 1959; 183:302-303.

15. Ford C. A sex-chromosome anomaly in a case of gonadal dysgenesis (Turner's syndrome). The Lancet. 1959; 273:711-713.

16. Duesberg P, McCormack A. Immortality of cancers: a consequence of inherent karyotypic variations and selections for autonomy. Cell Cycle Georget Tex. 2013; 12:783-802.

17. Duesberg P, Rausch C, Rasnick D, et al. Genetic instability of cancer cells is proportional to their degree of aneuploidy. Proc Natl Acad Sci USA. 1998; 95:13692-13697.

18. Nicholson JM, Duesberg P. On the karyotypic origin and evolution of cancer cells. Cancer Genet Cytogenet. 2009; 194:96-110.

19. Birchler JA. Aneuploidy in plants and flies: the origin of studies of genomic imbalance. Semin Cell Dev Biol. 2013; 24:315-319.

20. Mank JE. Sex chromosome dosage compensation: definitely not for everyone. Trends Genet. 2013; Epub. doi:10.1016/j.tig.2013.07.005

21. Bull JJ. Evolution of Sex Determining Mechanisms. San Fransico, USA: Benjamin Cummings; 1983.

22. Georges A, Ezaz T, Quinn AE, et al. Are reptiles predisposed to temperature-dependent sex determination? Sex Dev Genet Mol Biol Evol Endocrinol Embryol Pathol Sex Determ Differ. 2010; 4:7-15.

23. Quinn AE, Sarre SD, Ezaz T, et al. Evolutionary transitions between mechanisms of sex determination in vertebrates. Biol Lett. 2011; 7:443-448.

24. McElreavey $\mathrm{K}$, Barbaux $\mathrm{S}$, Ion $\mathrm{A}$, et al. The genetic basis of murine and human sex determination: a review. Heredity (Edinb). 1995; 75 (Pt 6):599-611.
25. Capel B. The role of Sry in cellular events underlying mammalian sex determination. Curr Top Dev Biol. 1996; 32:1-37.

26. Sinclair $\mathrm{AH}$, Berta $\mathrm{P}$, Palmer MS, et al. A gene from the human sex-determining region encodes a protein with homology to a conserved DNA-binding motif. Nature. 1990; 346:240-244.

27. Herpin A, Schartl M. Dmrt1 genes at the crossroads: a widespread and central class of sexual development factors in fish. FEBS J. 2011; 278:1010-1019.

28. Robinett CC, Vaughan AG, Knapp J-M, et al. Sex and the single cell. II. There is a time and place for sex. PLoS Biol. 2010; 8:e1000365.

29. Clough E, Oliver B. Genomics of sex determination in Drosophila. Briefings Funct Genomics. 2012; 11:387-394.

30. Hildreth Pe. Doublesex, Recessive Gene That Transforms Both Males And Females Of Drosophila Into Intersexes. Genetics. 1965; 51:659-678.

31. Shen MM, Hodgkin J. mab-3, a gene required for sex-specific yolk protein expression and a male-specific lineage in C. elegans. Cell. 1988; 54:1019-1031.

32. Raymond CS, Murphy MW, O'Sullivan MG, et al. Dmrt1, a gene related to worm and fly sexual regulators, is required for mammalian testis differentiation. Genes Dev. 2000; 14:2587-2595.

33. Raymond CS, Shamu CE, Shen MM, et al. Evidence for evolutionary conservation of sex-determining genes. Nature. 1998; 391:691-695.

34. Chue J, Smith CA. Sex determination and sexual differentiation in the avian model. FEBS J. 2011; 278:1027-1034.

35. Shoemaker C, Ramsey M, Queen J, et al. Expression of Sox9, Mis, and Dmrt1 in the gonad of a species with temperature-dependent sex determination. Dev Dyn Off Publ Am Assoc Anat. 2007; 236:1055-1063.

36. Yoshimoto S, Okada E, Umemoto $\mathrm{H}$, et al. A W-linked DM-domain gene, DM-W, participates in primary ovary development in Xenopus laevis. Proc Natl Acad Sci USA. 2008; 105:2469-2474.

37. Bridges CB. Sex in relation to chromosomes and genes. Am Nat. 1925; 59:127-137.

38. Erickson JW, Quintero JJ. Indirect effects of ploidy suggest X chromosome dose, not the X:A ratio, signals sex in Drosophila. PLoS Biol. 2007; 5:e332.

39. Haag E. The evolution of nematode sex determination: C. elegans as a reference point for comparative biology. WormBook. 2005.

40. Green DM. Cytogenetics of the endemic New Zealand frog, Leiopelma hochstetteri: extraordinary supernumerary chromosome variation and a unique sex-chromosome system. Chromosoma. 1988; 97:55-70.

41. Felsenstein J. The evolutionary advantage of recombination. Genetics. 1974; 78:737-756.

42. Charlesworth B. Model for evolution of $\mathrm{Y}$ chromosomes and dosage compensation. Proc Natl Acad Sci. 1978; 75:5618-5622.

43. Rice WR. Sex Chromosomes and the Evolution of Sexual Dimorphism. Evolution. 1984; 38:735-742.

44. Rice WR. The Accumulation of Sexually Antagonistic Genes as a Selective Agent Promoting the Evolution of Reduced Recombination between Primitive Sex Chromosomes. Evolution. 1987; 41:911-914.

45. Fisher RA. The evolution of dominance. Biol Rev. 1931; 6:345-368.

46. Bachtrog D. Y-chromosome evolution: emerging insights into processes of Y-chromosome degeneration. Nat Rev Genet. 2013; 14:113-124.

47. Larsson J, Meller VH. Dosage compensation, the origin and the afterlife of sex chromosomes. Chromosome Res Int J Mol Supramol Evol Asp Chromosome Biol. 2006; 14:417-431.

48. Engreitz JM, Pandya-Jones A, McDonel P, et al. The Xist lncRNA Exploits Three-Dimensional Genome Architecture to Spread Across the X Chromosome. Science. 2013; 341:1237973.

49. Plath K, Mlynarczyk-Evans S, Nusinow DA, et al. Xist RNA and the Mechanism of X Chromosome Inactivation. Annu Rev Genet. 2002; 36:233-278.

50. Sun L, Johnson AF, Li J, et al. Differential effect of aneuploidy on the $\mathrm{X}$ chromosome and genes with sex-biased expression in Drosophila. Proc Natl Acad Sci USA. 2013; 110:16514-16519.

51. Larschan E, Bishop EP, Kharchenko PV, et al. X chromosome dosage compensation via enhanced transcriptional elongation in Drosophila. Nature. 2011; 471:115-118

52. Soruco MML, Chery J, Bishop EP, et al. The CLAMP protein links the MSL complex to the $\mathrm{X}$ chromosome during Drosophila dosage compensation. Genes Dev. 2013; 27:1551-1556.

53. Zhang Y, Malone JH, Powell SK, et al. Expression in aneuploid Drosophila S2 cells. PLoS Biol. 2010; 8:e1000320.

54. Livernois AM, Waters SA, Deakin JE, et al. Independent evolution of transcriptional inactivation on sex chromosomes in birds and mammals. PLoS Genet. 2013; 9:e1003635.

55. Arnold AP, Itoh Y, Melamed E. A bird's-eye view of sex chromosome dosage compensation. Annu Rev Genomics Hum Genet. 2008; 9:109-127. 
56. Vicoso B, Emerson JJ, Zektser Y, et al. Comparative sex chromosome genomics in snakes: differentiation, evolutionary strata, and lack of global dosage compensation. PLoS Biol. 2013; 11:e1001643.

57. Vicoso B, Bachtrog D. Lack of global dosage compensation in Schistosoma mansoni, a female-heterogametic parasite. Genome Biol Evol. 2011; 3:230-235.

58. Wolf JB, Bryk J. General lack of global dosage compensation in ZZ/ZW systems? Broadening the perspective with RNA-seq. BMC Genomics. $2011 ; 12: 91$.

59. Devlin RH, Nagahama Y. Sex determination and sex differentiation in fish: an overview of genetic, physiological, and environmental influences. Aquaculture. 2002; 208:191-364.

60. Evans BJ, Pyron RA, Wiens JJ. Polyploidization and Sex Chromosome Evolution in Amphibians. In Polyploidy Genome Evol. Berlin, Germany: Springer; 2012:385-410.

61. Kopp A. Dmrt genes in the development and evolution of sexual dimorphism. Trends Genet. 2012; 28:175-184.

62. David M. Hillis DMG. Evolutionary changes of heterogametic sex in the phylogenetic history of amphibians. J Evol Biol. 2002; 3:49 - 64.

63. Miura I, Ohtani H, Nakamura M, et al. The origin and differentiation of the heteromorphic sex chromosomes $\mathrm{Z}, \mathrm{W}, \mathrm{X}$, and $\mathrm{Y}$ in the frog Rana rugosa, inferred from the sequences of a sex-linked gene, ADP/ATP translocase. Mol Biol Evol. 1998; 15:1612-1619.

64. Miura I. An evolutionary witness: the frog Rana rugosa underwent change of heterogametic sex from XY male to ZW female. Sex Dev Genet Mol Biol Evol Endocrinol Embryol Pathol Sex Determ Differ. 2007; 1:323-331.

65. Uno $Y$, Nishida C, Oshima $Y$, et al. Comparative chromosome mapping of sex-linked genes and identification of sex chromosomal rearrangements in the Japanese wrinkled frog (Rana rugosa, Ranidae) with ZW and XY sex chromosome systems. Chromosome Res Int J Mol Supramol Evol Asp Chromosome Biol. 2008; 16:637-647.

66. Rodrigues N, Betto-Colliard C, Jourdan-Pineau $\mathrm{H}$, et al. Within-population polymorphism of sex-determination systems in the common frog (Rana temporaria). J Evol Biol. 2013; 26:1569-1577.

67. Eggert C. Sex determination: the amphibian models. Reprod Nutr Dev. 2004; 44:539-549.

68. Schmid M, Steinlein C, Bogart JP, et al. The chromosomes of terraranan frogs. Insights into vertebrate cytogenetics. Cytogenet Genome Res. 2010; 130-131:1-568.

69. Perrin N. Sex reversal: a fountain of youth for sex chromosomes? Evol Int J Org Evol. 2009; 63:3043-3049.

70. Stöck M, Savary R, Zaborowska A, et al. Maintenance of ancestral sex chromosomes in Palearctic tree frogs: direct evidence from Hyla orientalis. Sex Dev Genet Mol Biol Evol Endocrinol Embryol Pathol Sex Determ Differ. 2013; 7:261-266.

71. Stöck M, Horn A, Grossen C, et al. Ever-young sex chromosomes in European tree frogs. PLoS Biol. 2011; 9:e1001062.

72. Stöck M, Savary R, Betto-Colliard C, et al. Low rates of X-Y recombination, not turnovers, account for homomorphic sex chromosomes in several diploid species of Palearctic green toads (Bufo viridis subgroup). J Evol Biol. 2013; 26:674-682.

73. Volff J-N, Nanda I, Schmid M, et al. Governing sex determination in fish: regulatory putsches and ephemeral dictators. Sex Dev Genet Mol Biol Evol Endocrinol Embryol Pathol Sex Determ Differ. 2007; 1:85-99.

74. Hotz H, Uzzell T, Berger L. Linkage groups of protein-coding genes in western palearctic water frogs reveal extensive evolutionary conservation. Genetics. 1997; 147:255-270.

75. Stöck M, Croll D, Dumas Z, et al. A cryptic heterogametic transition revealed by sex-linked DNA markers in Palearctic green toads. J Evol Biol. 2011; 24:1064-1070.

76. Malone JH, Fontenot BE. Patterns of reproductive isolation in toads. PLoS One. 2008; 3:e3900.

77. O'Meally D, Ezaz T, Georges A, et al. Are some chromosomes particularly good at sex? Insights from amniotes. Chromosome Res Int J Mol Supramol Evol Asp Chromosome Biol. 2012; 20:7-19.

78. Brelsford A, Stöck M, Betto-Colliard C, et al. Homologous sex chromosomes in three deeply divergent anuran species. Evol Int J Org Evol. 2013; 67:2434-2440.

79. Schmid M. Chromosome banding in Amphibia. V. Highly differentiated ZW/ZZ sex chromosomes and exceptional genome size in Pyxicephalus adspersus (Anura, Ranidae). Chromosoma. 1980; 80:69-96.

80. Wells KD. The Ecology \& Behavior of Amphibians. Chicago, USA: University of Chicago Press; 2007.

81. De Almeida CG, Grafe TU, Guttenbach M, et al. Karyotype and chromosome banding in the reed frog Hyperolius viridiflavus ommatostictus (Amphibia, Anura, Hyperoliidae). Experientia. 1990; 46:509-511.
82. Schmid M, Klett R. Chromosome banding in Amphibia. XX. DNA replication patterns in Gastrotheca riobambae (Anura, Hylidae). Cytogenet Cell Genet. 1994; 65:122-126.

83. Schmid M, Haaf T, Geile B, et al. Chromosome banding in Amphibia. VIII. An unusual XY/XX-sex chromosome system in Gastrotheca riobambae (Anura, Hylidae). Chromosoma. 1983; 88:69-82.

84. Ohta S, Sumida M, Nishioka M. Sex-determining mechanism in Buergeria buergeri (Anura, Rhacophoridae). III. Does the ZZW triploid frog become female or male? J Exp Zool. 1999; 283:295-306.

85. Ohta, S. Sex Determining Mechanism in Buergeria buergeri (SCHLEGEL) : I. Heterozygosity of Chromosome Pair No. 7 in the Female. Sci Rep Lab Amphib Biol Hiroshima Univ. 1986; 8:29-43.

86. Ohta S, Sumida M, Nishioka M. Sex-determining mechanism in Buergeria buergeri (Anura, Rhacophoridae). III. Does the ZZW triploid frog become female or male? J Exp Zool. 1999; 283:295-306.

87. Schmid M, Ohta S, Steinlein C, et al. Chromosome banding in Amphibia. XIX. Primitive ZW/ZZ sex chromosomes in Buergeria buergeri (Anura, Rhacophoridae). Cytogenet Cell Genet. 1993; 62:238-246.

88. Hayes TB. Sex determination and primary sex differentiation in amphibians: genetic and developmental mechanisms. J Exp Zool. 1998; 281:373-399.

89. Nascimento J, Quinderé YRSD, Recco-Pimentel SM, et al. Heteromorphic $\mathrm{Z}$ and W sex chromosomes in Physalaemus ephippifer (Steindachner, 1864) (Anura, Leiuperidae). Genetica. 2010; 138:1127-1132.

90. Schmid M, Feichtinger W, Steinlein C, et al. Chromosome banding in Amphibia. XXVI. Coexistence of homomorphic XY sex chromosomes and a derived Y-autosome translocation in Eluetherodactylus maussi (Anura, Leptodactylidae). Cytogenet Genome Res. 2002; 99:330-343.

91. Schmid M, Steinlein C, Feichtinger W. Chromosome banding in Amphibia. XVII. First demonstration of multiple sex chromosomes in amphibians: Eluetherodactylus maussi (Anura, leptodactylidae). Chromosoma. 1992; 101:284-292.

92. Kobel HR, du Pasquier L. Genetics of polyploid Xenopus. Trends Genet. 1986; 2:310-315.

93. Schmid M, Feichtinger W, Steinlein C, et al. Chromosome banding in amphibia. XXIII. Giant W sex chromosomes and extremely small genomes in Eluetherodactylus euphronides and Eluetherodactylus shrevei (Anura, Leptodactylidae). Cytogenet Genome Res. 2002; 97:81-94.

94. Schmid M, Steinlein C, Friedl R, et al. Chromosome banding in Amphibia. XV. Two types of $\mathrm{Y}$ chromosomes and heterochromatin hypervariabilty in Gastrotheca pseustes (Anura, Hylidae). Chromosoma. 1990; 99:413-423.

95. Schmid M, Steinlein C, Bogart JP, et al. The hemiphractid frogs. Phylogeny, embryology, life history, and cytogenetics. Cytogenet Genome Res. 2012; 138:69-384.

96. Stancheva I, Meehan RR. Transient depletion of xDnmt1 leads to premature gene activation in Xenopus embryos. Genes Dev. 2000; 14:313-327.

97. Liu E, Pott S, Huss M. Q\&A: ChIP-seq technologies and the study of gene regulation. BMC Biol. 2010; 8:56.

98. Chang CY, Witschi E. Genic control and hormonal reversal of sex differentiation in Xenopus. Proc Soc Exp Biol Med Soc Exp Biol Med New York N. 1956; 93:140-144.

99. Mikamo K, Witschi E. Functional sex-reversal in genetic females of Xenopus laevis, induced by implanted testes. Genetics. 1963; 48:1411-1421.

100. Malone JH, Michalak P. Physiological sex predicts hybrid sterility regardless of genotype. Science. 2008; 319:59. 